\title{
Which Female Prefer Longer Penises?
}

\author{
Kim M. DRASA Jr. ${ }^{1}$, Vaso Vasili ${ }^{2}$ \\ Central Polyclinic, Tirana, Albania
}

\begin{abstract}
Introduction: An orgasm in the human female is a transient peak sensation of intense pleasure creating an altered state of consciousness, usually with an initiation accompanied by involuntary, rhythmic contractions of the pelvis striated musculature,often with concomitant uterine and anal contractions and myotonia that resolves the sexually-induced vaso congestion and usually with an induction of well-being and contentment. Women's orgasms can be induced by erotic stimulation of a variety of genital and non-genital sites. Women`s orgasms are four kind: 1) Clitoridene, 2) G-spot , 3) Vaginal, 4) All three together. Lack of orgasm is the second most common female sexual problem, affecting more than $20 \%$ of woman. Aim: The aim of our study was to find if the vaginal orgasm provoke only when penis of her partner is longer than average penis. Material and methods: The study was an online survey and by in person surveys. The present sample consist of 1275 coitally experienced women. The effect of penis size on the likelihood of having an orgasm from penile - vaginal intercourse (PVI), was assessed by the questions :" Are you more likely to have orgasm from penis-invagina intercourse with a man who has somewhat longer than average penis"?. (Assume that average erect penis length is 149-155 $\mathrm{m} / \mathrm{m}$ long).Possible answers were: a) More likely to have an orgasm with longer penis, b) No difference - I orgasm equally well with long or average penis lengths; c) Less likely to have an orgasm with a longer penis; d) I do not (or do not often) have an orgasm from intercourse; or e) I have not had enough penis-in-vagina intercourse partners to make a comparison.(The £20 note is $149 \mathrm{~m} / \mathrm{m}$ long and $U$;S; dollar bills are $155 \mathrm{~m} / \mathrm{m}$ long). Exclusion criteria were being coitally inexperienced and responses suggesting a high risk of misreporting, as indicated by scores the $86^{\text {th }}$ percentile on the short version of the Marlowe - Crowne Social Desirability Scale (the 13item short form). Results: Women's reports on how penis size influences the likelihood of reaching orgasm from PVI were: a) More likely to have an orgasm with a longer penis-35.7\%; b) No-difference-equally likely of having an orgasm with a longer and average penis $56.5 \%$; c) Less likely to have an orgasm with a longer penis 2,1\%; d) Never had an orgasm from PVI-1.4\%; e) Not enough PVI partners to make a comparison $4.3 \%$. Women who reported that they were more likely to reach orgasm from PVI with a longer than average penis reported having more vaginal orgasms within a previous month compared with women for whom penis size is not important for PVI orgasm. The likelihood of attaining orgasm from PVI with a longer that average penis was independently predicted by greater importance attributed to PVI and lesser importance attributed to noncoital sex: OR (95\%CI), p<0.05. Conclusion: Women who prefer deeper penile-vaginal stimulation are more likely to have vaginal orgasm, consistent with vaginal orgasm evolving as part of the female mate choice system favouring somewhat longer that average penis. Like many men's desire to have a longer than average penis and the desire of (especially vaginal orgasmic) women is to have a man with a longer than average penis. Penis size appears to be important for many women. Conclusion by in-personal survey seemed to be similar with my colleagues but, for me, with one addition: "Penis for both likelihood of orgasm (women / men) is best to be strong as a rock than to be large but not strong".
\end{abstract}

Keywords: Penis, women, orgasm

\section{Introduction}

Through the years, different models have been proposed to explain sexual responding. The latter is nevertheless important because sex often takes place in the context of a relationship and is thus strongly influenced by partner responses and relational features (1-3).

The penis particularly in its erect state is a symbol of masculinity. In many cultures it has come to symbolize attributes such as strength, virility, possession and courage. The size of the penis is therefore an important aspect of life in the male from puberty to adulthood. Throughout history, there have been a variety of references to penile enhancement size. The Indian Sadhus and Peruvian Cholomecs used weights, similar to the Penile Tissue Expander to attain increased lengths of 12 to 18 inches. In the $16^{\text {th }}$ century, the Topinama of Brazil would significantly enlarge their penis by encouraging poisonous snakes to bite their penis. At the end of six months the men had a monstruous-sized penis that delighted their woman.

In the Diagnostic and Statistical Manual of Mental Disorders, $5^{\text {th }}$ Edition (DSM -5; F52.31), four diagnostic criteria must be met in order to classify as Female Orgasmic Disorder
(FOD),(4). According to the DSM - 5, FOD can be categorized into various subtypes including lifelong vs acquired and generalized vs situational FOD,(4).

Very often in literature, orgasm problems are referred to as either "primary" or "secondary".

The definition of orgasm itself remains vogue, which makes it difficult for clinicians to determine a diagnosis. Because the exact mechanism of the cerebral neuronal discharge is so poorly understood, most definition of orgasm, in 2003 an International Consultation, in collaboration, assembled a large group of experts who agreed on the following definition:

"A variable, transient peak sensation of intense pleasure creating an altered state of consciousness, usually accompanied by involuntary, rhythmic contractions of the pelvic striated circum vaginal musculature, often with concomitant uterine and anal contractions and myotonia that resolves the sexually induced vasocongestion (sometimes only partially), usually with an induction of well-being and contentment".

When woman have sex with men, their sexual pleasure is influenced by the male partner's sexual performance, as well as by his physical and psychological characteristics. Research 


\section{International Journal of Science and Research (IJSR) \\ ISSN (Online): 2319-7064 \\ Index Copernicus Value (2013): 6.14 | Impact Factor (2015): 6.391}

also shows that male traits such as penis size and copulatory vigor are more relevant for woman'sattain orgasm from penile-vaginal intercourse (PVI) than from other sexual activities. The likelihood of a woman attaing an orgasm through penile stimulation of the vagina without concurrent clitoral masturbation (hence forth vaginal orgasm) is related to men's erectile function (5) and to duration of PVI, but not to duration of foreplay (in multivariate analyses controlling for both PVI and foreplay duration),(6).

Our theory, linking greater likelihood of vaginal orgasm to having a man with a longer penis, is a special case of the evolutionary view that female orgasm evolved as a mate choice $\operatorname{system}(7)$ and is consistent with the view that vaginal orgasm can be a signal of greater fitness of both partners,(8). Increasing evidence shows that penis size is important for sexual pleasure of many women and is arguably more relevant during PVI than during other sexual activities. There is evidence that the entire length of the vagina (and cervix) is well innervated and that (in addition to an overlapping general region) the cervix projects to a different region of the somatosensory cortex than the distal vagina, which in turn projects to a different region than the clitoris, $(9.10)$.

In additional, substantial evidence shows that most women care about penis size to some degree and typically prefer a somewhat thicker and longer penis than average.

Finally, if preference for a longer penis is driven by greater vaginal-cervical sensitivity, then one might infer that women who reach orgasm from PVI more easily with longer penises value PVI relatively more and value noncoital sex relatively less.

\section{Aim}

The aim of our study was to find if the vaginal orgasm provoke only when penis of her partner is longer than average penis.

\section{Method}

The study was an online survey. The quality of sexuality date collected by online survey appears to be similar and by inpersonal survey. The present sample consist on 1275 coitally experienced women. The effect of penis size on the likelihood of having an orgasm from PVI was assessed by the question:

"All things being equal, are you more likely to have an orgasm from penis -in -vagina intercourse with a man who has a somewhat larger than average penis length?

(Assume that average erect penis length is the length of a L20 note or any U.S dollar bill)".

Possible answers were: (a) more likely to have an orgasm with a longer penis, (b) no difference - I orgasm equally well with a long or average penis length, (c) less likely to have an orgasm with a longer penis, (d) I do not (or do not often) have an orgasm from intercourse, or(e) I have not had enough penis- in- vagina intercourse partners to make a comparison (the L 20 note is $149 \mathrm{~m} / \mathrm{m}$ long and U.S. dollar bills are $155 \mathrm{~m} / \mathrm{m}$ long)

Exclusion criteria were being coitally inexperienced and responses suggesting a high risk of misreporting, as indicated by scores the $86^{\text {th }}$ percentile on the short version of the Marlowe-Crowne Social Desirability Scale,(11).

Participants reported how many days they engaged in the following sexual behaviors and how many days they had orgasms from each of them in a recent representative month: PVI without additional simultaneous clitoral stimulation, clitorally focused masturbation (except vibrator), vaginally focused masturbation (except vibrator ), masturbation with vibrator (alone or with a partner), clitorally focused manual stimulation by a partner, receiving oral sex and anal sex. Clitorally focused masturbation, vaginally focused masturbation, oral sex, and anal sex were differentiated as occurring with or without PVI in the same day, $(12,13,14,15)$.

\section{Statistical Analysis}

Were used univariate analyses of covariance to compare women who reported that they were more likely to attain orgasm from PVI with a longer than average penis to women for whom penis size was not important, with respect to frequencies of various sexual behaviors including orgasms from each behavior. All statistical analyses were performed with SPSS, version 17 (SPSS Inc., Chicago, IL, USA).

\section{Results}

Women's reports on how penis size influences the likelihood of reaching orgasm from penile-vaginal-intercourse (PVI) are as below:

- More likely to have orgasm with a longer penis were $=$ $35.7 \%$

- No -difference - equally likely of having an orgasm with a longer penis were $=56.5 \%$

- Less likely to have an orgasm with a longer penis $=2 \%$

- Never had an orgasm from PVI $=1.4 \%$

- Not enough PVI partners to make a comparison $=4.3 \%$

Women who reported that they were more likely to reach orgasm from PVI with a longer than average penis reported having more vaginal orgasms within a previous month compared with women for whom penis size is not important for PVI orgasm. Notably, the groups do not differ in the frequencies of clitoral orgasm, orgasm induced by vaginal masturbation, or any other sexual behaviors.

The likelihood of attaining orgasm from PVI with a longer than average penis and so were independently predicated by greater importance attributed to PVI and lesser importance attributed to noncoital sex: OR $(95 \% \mathrm{CI})$

\section{Discussion}

This study replicated and extends earlier findings(6) by showing that the importance women give to penis size for 


\section{International Journal of Science and Research (IJSR) \\ ISSN (Online): 2319-7064}

Index Copernicus Value (2013): 6.14 | Impact Factor (2015): 6.391

reaching orgasm from PVI is associated with higher frequency of orgasm from vaginal masturbation, frequency of clitoral orgasm, or frequency of any other sexual activities. Higher vaginal cervical responsiveness among women who can more easily attain vaginal orgasm might be related, at least in part, to greater thickness and/or length of the urethra vaginal space $(16,17)$, better vagus nerve function $(15,18)$ more awareness of vaginal erotic responses $(19,20)$, more attention to vaginal sensations during PVI (6), better pelvic region muscle function (21), better psychological functioning in at least some respects $(5,12,14,22,23,24,25)$, better intimate relationship quality $(5,22,25,26)$, and higher sexual desire $(25,31)$

One might argue that more generally erotophilic or less inhibited women rate larger penises as more important and also have higher rates of all sexual activities and all kinds of orgasm.

However, our differential results argue against that interpretation because the preference for a longer penis is associated only with vaginal orgasm frequency, not with frequency of other forms of orgasm or other sexual activities. Our results might slightly underestimate women's true size preferences because their preferences for an" average" penis length ( the $£ 20$ note at $149 \mathrm{~m} / \mathrm{m}$ long and the U.S. dollar bill at $155 \mathrm{~m} / \mathrm{m}$ long) are slightly longer than the approximately $148 \mathrm{~m} / \mathrm{m}$ mean erect penis length found when averaging results across several studies.

Vaginal orgasm (or orgasm during PVI) is more likely to occur when women and/ or their partners show specific indicators of mate value such as:

- Better physical or psychological health $(5,8,12,14,15,22,23,25,27)$.

- When their male partners have other indices of mate value, such as higher physical attractiveness $(28,29)$, masculinity (28), body symmetry (30) and copulatory courtship ability (indicated by greater erectile potency and lo9nger PVI duration $(5,6)$ and

- When couples find themselves in more reproductively propitious relationships, as cued by higher sexual desire, higher sexual satisfaction, and higher relationship satisfaction $(5,8,22,25,26,31)$.

Consistent with vaginal orgasm having evolved to promote desire for PVI in favorable circumstances.

We think that vaginal orgasm and PVI frequency are associated with greater sexual desire and passion (25,26,31), sexual satisfaction $(5,25,32,33,34)$, and less risk of sexual dysfunctions (31,35 36), whereas other sexual activities are not as clearly related to better sexual function; indeed, they are often unrelated or correlated with impaired sexual function $(2,31,32,33,34,35)$

Our plausible explanation for the relationship between female PVI orgasm and male attributes is the so-called "cryptic female mate choice "that is, female preferences expressed during copulation that affect likelihood of fertilization and/ or of remating with the same male. Cryptic female choice has shaped the evolution of male intromittent genitalia and copulatory behavior across many species (36).

These correlational findings can be a useful guide for informing futurecontrolled clinical research studies.

\section{Conclusion}

Women's frequency of vaginal orgasm is associated with preference for deeper penile-vaginal stimulation, as indicated by greater importance given to longer than average penises. In contrast to the assertions common in sexology, penis size appears important to many women, and this is consistent with evolutionary hypotheses concerning the mate choice functions of vaginal orgasm and with the biological literature showing cryptic female choice for male genital morphology across many species. Like many men's desire to have a longer than average penis, the desire of (especially vaginally orgasmic) women to have a man with a longer than average penis may reflect lessons learned from real sexual experiences rather than internalization of arbitrary stereotypes

Conclusion by in-personal survey seemed to be similar with my colleagues but, for me, with one addition: "Penis for both likelihood of orgasm (women / men) is best to be strong as a rock than to be large but not strong".

\section{References}

[1] Dewitte M. On the interpersonal dynamics in sexuality. J Sex MaritalTher 2014; 40: 209-32.

[2] Birnbaum GE, Laser-Brandt D. Gender differences in the experience of heterosexual intercourse. Can J Hum Sex 2000; 11: 143-58.

[3] Pakpour AH, Yakininejad MS, Pallich G, Burri A. Using ecological momentary assessment to investigate short-term variations in sexual functioning in a sample of peri-menopausal women from Iran. PLoS ONE 2015; 10: $1-14$.

[4] American Psychiatric Association. Diagnostic and Statistical Manual of Mental Disorders. $5^{\text {th }}$ ed. Arlington, VA: American Psychiatric Publishing; 2013. P. 5-25.

[5] Weiss P, Brody S. International Index of Erectile Function(IIEF) scores generated by men or female partners correlate equally well with own satisfaction (sexual, partnership, life, and mental health). J Sex Med 2011; 8: 1404-10.

[6] Brody S, Weiss P. Vaginal orgasm is associated with vaginal (not clitoral) sex education, focusing mental attention on vaginal sensations, intercourse duration, and a preference for a longer penis. J Sex Med 2010; 7: 2774-81.

[7] Puts DA, Dawood K, Welling LL. Why women have orgasms: An evolutionary analysis. Arch Sex Behav 2012 jun 26 (Epub ahead of print) DOI 10.1007/ s 10508-012-9967-x.

[8] Brody S, Costa RM, Hess U, Weiss P. Vaginal is related to better menthal health and is relevant to evolutionary 


\section{International Journal of Science and Research (IJSR) \\ ISSN (Online): 2319-7064}

Index Copernicus Value (2013): 6.14 | Impact Factor (2015): 6.391

psychology: A response to Zietsch et al. J Sex Med 20011; 8: 3523-5.

[9] Pauls R, Mutema G ,Segal J, Silva WK, Kleeman S, Dryghout $\mathrm{Ma} \mathrm{V}$, Karram M. A prospective study examining the anatomic distribution of nerve density in human vagina. J Sex Med 2006; 3: 979-87.

[10] Komisaruk BR, Wise N, Frangos E, Liu W-C, Allen K, Brody S. Women's clitoris, vagina and cervix mapped on the sensory cortex: fMRI evidence. J Sex Med 2011, 8: 2822-30.

[11] Ballard R. Short forms of the Marlowe-Crowne Social Desirability Scale. Psychol Rep 1992; 71: 1155-60.

[12] Costa RM, Brody S. Immature defense mechanisms are associated with lesser vaginal orgasm consistency and greater alcohol consumption before sex. J Sex Med 2010; 7: 775-86.

[13] Brody S, Costa RM. Overestimation of heterosexually attributed AIDS deaths is associated with immature psychological defence mechanisms and clitoral masturbation during penile-vaginal intercourse. Int $\mathrm{J}$ STD AIDS 2009; 20: 869-75.

[14] Costa RM, Brody S. Anxious and avoidant attachment, vibrator use, anal sex, and impaired vaginal orgasm. J Sex Med 2011; 8. 2493-500.

[15] Costa RM, Brody S. Greater resting heart rate variability is associated with orgasms through penilevaginal intercourse but not with orgasms other sources. J Sex Med 2012; 9:188-97.

[16] Gravina GL, Brandetti F, Martini P, Carosa E, Di Stassi SM, Morano S, Lenzi A, Jannini EA. Measurement of the thickness of the urethrovaginal space in women with or without vaginal orgasm. J Sex Med 2008, 5: 610-8.

[17] Battaglia C, nappi RE, Mancini F, Alvisi S, Del Forno S, Battaglia C, Venturoli S. 3-D volumetric and vascular analysis of the uretrovaginal space in young women with or without vaginal orgasm. J Sex Med 2010; 7: 1445-53.

[18] Brody S, Preut R. Vaginal intercourse frequency and heart rate variability. J Sex Marital Ther 2003; 29: 37180 .

[19] Brody S. Intercourse orgasm consistency, concordance of women's genital and subjective sexual arousal, , and erotic stimulus presentation sequence. J Sex Marital Ther 2007; 33: 31-9.

[20] Brody S, laan E, van Lunsen RWH. Concordance between women's physiological and subjective sexual arousal is associated with consistency of orgasm during intercourse but not other sexual behaviour. J Sex Marital Ther 2003, 29: 15-23.

[21] Nicholas A, Brody S, de Sutter P, de Carufel F. A woman's history of vaginal orgasm is discernible from her walk. J Sex Med 2008; 5: 2119-24.

[22] Brody S. The relative health benefits of different sexual activities. J Sex Med 2010; 7: 1336-61.

[23] Brody S, Costa RM. Vaginal orgasm is associated with less use of immature psychological defense mechanisms. J Sex Med 2008; 5: 1167-76.

[24] Costa RM, Brody S. Condom use for penile-vaginal intercourse is associated with immature psychological defensemechsnisms. J Sex Med 2008; 5: 2522-32.
[25] Brody S. Vaginal orgasm is associated with better psychological functioning. Sex RelatTher 2007; 22: 173-91.

[26] Costa RM, Brody S. Women's relationship quality is associated with specifically penile- vaginal intercourse orgasm and frequency. J Sex Marital Ther 2007; 33: 319-27.

[27] Awwad Z, Abu-Hijleh M, Basri S, Shegam N, Murshidi M, Ajlouni K. Penile measurements in normal adult Jordanians and in patients with erectile dysfunction. Int J Impot Res 2005; 17: 191-5.

[28] Shackelford TK, Weeks-Shackelford VA, LeBlane GJ, Bleske AL, Euler HA, Hoier S. Female coital orgasm and male attractiveness. Hum Nat 2000; 11: 299-306.

[29] Puts DA, Welling LLM, Burriss RP, Dawood K. Men's masculinity and attractiveness predict their female partners' reported orgasm frequency and timing. Evol Hum Behav 2011; 33: 1-9.

[30] Thornhill R, Gangestad SW, Comer R. Human female orgasm and mate fluctuating asymmetry. AnimBehav 1995; 50. 1601-15.

[31] Nutter DE, Condrom MK. Sexual fantasy and activity patterns of females with inhibited sexual desire versus normal controls. J Sex Marital Ther 1983; 9: 276-82.

[32] Brody S costa RM. Satisfaction (sexual,life,relationship, and mental health) is associated directly with penilevaginal intercourse but inversely with other sexual behaviour frequencies. J Sex med 2009; 6: 1947-54.

[33] Philippsohn S, Hartmann U. Determinants of sexual satisfaction in a sample of German women. J Sex Med 2009; 6: 1001-10.

[34] Tao P, Brody S. Sexual behaviour predictors of satisfaction in a Chinese sample. J Sex Med 2011; 8: 455-60.

[35] Weiss P, Brody S. Female sexual arousal disorder with and without a distress criterion: Prevalence and correlates in a representative Czech sample. J sex med 2009; 6: 3385-94.

[36] Eberhard WG. Evolution of genitalia: Theories, evidence, and new directions. Genetica 2010; 138: 518. 